\title{
REVIEW
}

\section{Proteomics and Bioinformatics as Novel Tools in Phytoremediation Technology- An Overview}

\author{
Monalisa Mohanty* \\ Department of Biotechnology, Laboratory of Environmental Biotechnology, Rama Devi Women's University, \\ Bhubaneswar, India
}

\section{ARTICLE INFO}

Article history

Received: 18 June 2021

Accepted: 30 June 2021

Published Online: 7 July 2021

\section{Keywords:}

Bioinformatics

Proteomics

Genomics

Phytoremediation

Transgenic plants

\begin{abstract}
Biotechnology plays an important role in mitigation of various pollution in a cost effective manner by using the complex chemistry of living organisms, various cell manipulations and their approaches for environmental cleanup along with environmental sustainability. One such technology is phytoremediation technology or green technology which has emerged and evolved as a novel tool for remediation of toxic contaminants from environment. Plants with its diverse range show a remarkable range of their phytoremediation potentiality for establishing a sustainable environment. There is a huge exploitation of natural resources through expanded industrialization, urbanization, modern agricultural development, energy generation to fulfill the never-ending human desires and need. This disturbs the balance in nature where we reside and leads to progressive deterioration of the environment. There are several biotechnological advances which are employed for combating both the biotic and abiotic stress problems caused due to toxic contaminants in the environment. Various biotechnological interventions such as bioinformatics, proteomics, genomics, metallomics and metabolomics play a crucial role and open new avenue in this context. This omics approach is now integrated with bioinformatics to serve as a novel tool in phytoremediation technology. This smart technology provides insights into the complex behavior of enzymes, proteins and metabolites action and their biochemical pathways for degradation of wastes. This leads towards deriving a sustainable solution for environmental pollution.
\end{abstract}

sources through expanded industrialization, urbanization, modern agricultural development, energy generation to fulfill the never-ending human desires and need. This disturbs the balance in nature where we reside and leads to progressive deterioration of the environment.

Biotechnology plays an important role in mitigation of various pollution in a cost effective manner by using the complex chemistry of living organisms, various cell manipulations and their approaches for environmental

*Corresponding Author:

Monalisa Mohanty,

Department of Biotechnology, Laboratory of Environmental Biotechnology, Rama Devi Women's University, Bhubaneswar, India;

Email: monalisamohanty@rdwu.ac.in 


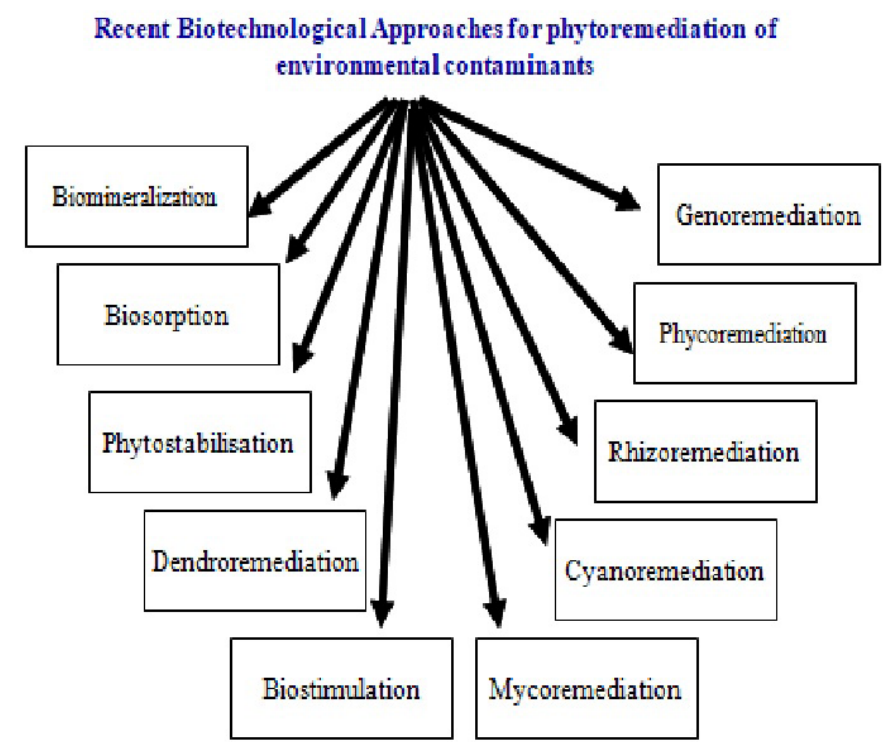

Figure 1. Biotechnological interventions for phytoremediation of environmental contaminants

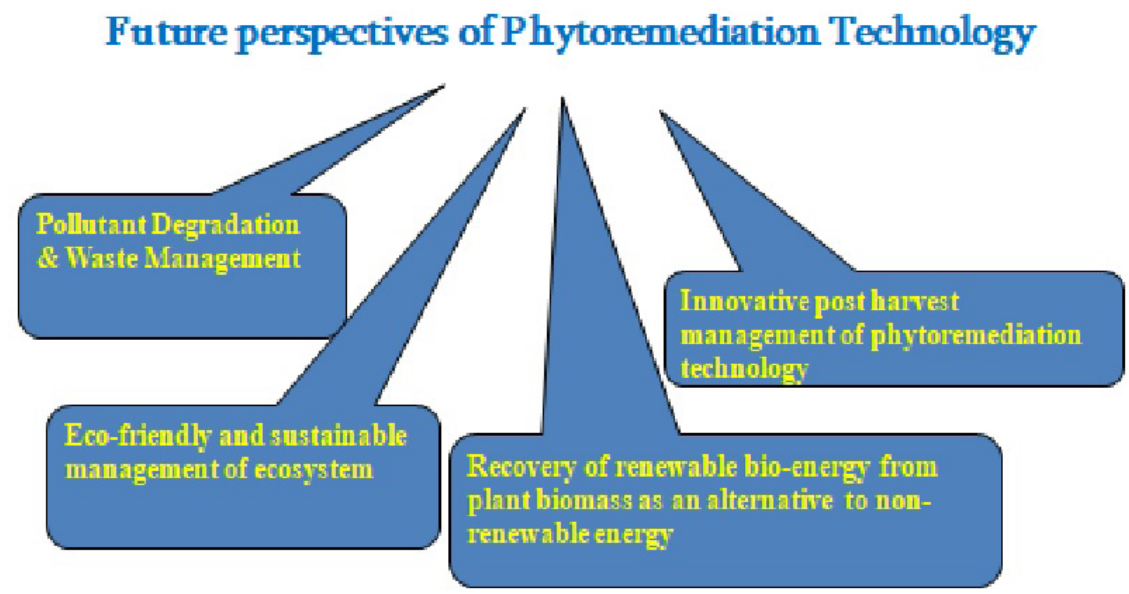

Figure 2. Future prospects of phytoremediation technology

cleanup along with environmental sustainability (Figure 1). One such technology is phytoremediation technology or green technology which has emerged and evolved as a novel tool for remediation of toxic contaminants from environment ${ }^{[1]}$ and emerges with a number of future perspectives (Figure 2). Various biotechnological interventions such as bioinformatics, proteomics, genomics, metallomics and metabolomics play a crucial role and open new avenue in this context. This omics approach is now integrated with bioinformatics to serve as a novel tool in phytoremediation technology.

\section{Role of Proteomics and Bioinformatics for Phytoremediation}

A variety of pollutants such as xenobiotics, polycyclic aromatic hydrocarbons (PAHs), heavy metals, chlorinated and nitro-aromatic compounds are persistent, highly toxic, mutagenic and carcinogenic for living organisms through bioaccumulation, adsorption and biotransformation ${ }^{[2,3]}$. A number of diverse and versatile plants, with high adaptability in the environment, are considered to be the potential tool among all living organisms to remediate most of the environmental contaminants. These natural agents can reduce wastes and cleanup the environmental contaminants by integrating with bioinformatics and proteomics.

The physiological changes in an organism during Phytoremediation can be best traced by using a proteomic approach, that further provide insight into remediation-related genes and their regulation ${ }^{[4]}$. Gene discovery through the use of computer software has developed advanced methods for the improvement of seed quality, stress tolerant transgenic plants, and engineer plants with phytoremediation capabilities. 
Detoxification of pollutants follows a path of uptake followed by metabolism and compartmentation in plants which are controlled by large families of genes and their expression. From expression profiles at the m- RNA level, proteomics provide information on where, when and at what level specific proteins accumulate in response to toxicants ${ }^{[5]}$.

Diverse group of plants show a remarkable range of their phytoremediation potentiality for establishing a sustainable environment. Contaminant induced protein expression studies as well as exploring the protein aggregation complexes like protein-protein interaction (interactomics) enables us for systematic study of plant physiological expressions in response to contaminants.

For example genetically, engineered plants (Arabidopsis thaliana) have been used as a tool of phytoextraction of arsenic pollutants and sequestration of As in vacuoles. These plants contain two bacterial genes of which one gene helps in conversion of arsenate into arsenite and the other binds to the arsenite and is finally stored in the vacuoles. In addition to this modified genes synthesizing different functional proteins (enzymes) involved in biochemical reactions in plants include phytoextraction, phytostabilization, phytotransformation, phytostimulation, and phytovolatilization played vital roles in remediation of contaminants ${ }^{[6]}$.

Wojas et al. ${ }^{[7]}$ demonstrated heterologous expression of AtMRP7 in modified Nicotiana tabacum var. Xanthi for higher cadmium accumulation, its distribution and tolerance in plants using Arabidopsis spp. MRP7 both in the tonoplast and in the plasma membrane of tobacco. The gene overexpression increased $\mathrm{Cd}$ tolerance and accumulation in tobacco leaf vacuoles, indicating more efficient detoxification by means of vacuolar storage. Heterologous AtMRP7 expression also led to more efficient retention of $\mathrm{Cd}$ in roots, with reduced root-to-shoot translocation.

The genes responsible for synthesis of proteins which help in uptake, sequestration, transportation and bioconcentration of different heavy metals as well as toxic organic and inorganic pollutants have been modified as per the desire which opens its avenue for efficiency of phytoremediation. Although intrinsic or natural phytoremediation is having more advantages than omics engineered phytoremediation, still application of omics based phytoremediation has gained attention of several researchers for stimulated removal of pollutants. Enhanced level of engineered proteins and enzymes will facilitate the mobilization, enhanced transportation in the root cell membrane and facilitate translocation to the shoot, modify, conjugate or degrade pollutants and facilitate tolerance, degradation, sequestration or volatilization. Several researchers inves- tigated the molecular genetic groundwork for enhanced metal tolerance through characterizing and identifying the genes encoding bacterial inorganic transformation ${ }^{[8-10]}$.

A number of plant species, such as tobacco, yellow poplar, cottonwood, and rice, expressing modified merA genes, have showed ten fold higher resistant to $\mathrm{Hg}$ (II) than non-transgenic plants ${ }^{[1]}$. Lyyra et al. ${ }^{[12]}$ reported that the both merA and merB genes in yellow poplar and cottonwood plants, for their increased potential for $\mathrm{Hg}$ phytovolatilization in wetlands. Genetic manipulation to yield metal tolerant plants, metalophore or hyperaccumulators for enhanced metal accumulation and tolerance through over-expression of natural or modified genes encoding antioxidant enzymes, or those that are involved in the biosynthesis of glutathione and other phytochelatins have been focused by several researchers and draws their attention. Banuelos et al. ${ }^{[13]}$ reported the efficiency of transgenic Brassica juncea plant with higher biomass and accumulation potential for $\mathrm{Se}$ and $\mathrm{Cd}$ than the wild type

Bioinformatics approach for phytoremediation is one such technique which has created a great revolution in management of huge integrated database created through genome expression, genome profiling, protein profiling, and expression level of proteins.

As per the report of Poirier et al. ${ }^{[14]} \mathrm{Cd}$ was the most toxic metal for Pseudomonas fluorescens BA3SM1, They showed that the bacterium is able to acquire a metal-resistant phenotype, making the strain BA3SM1 a promising agent for bioremediation processes.

In the recent era Phytoremediation confronts a number of challenges, which needs establishment of efficient integration between different academic areas such as plant physiology, biochemical pathway of contaminant uptake and translocation, biotechnology, ethnobiology, metallomics, genomics, metabollomics and proteomics; improved research practice; practical based experiences as interpretation of results produced and the applied methodologies; and the progress in monitoring programs based on environmental pollution.

Omics based phytoremediation adds value to the processes of phytomining, bioenergy, biomineralization, biostimulation, mycoremediation, cyanoremediation, phytodegradation, phytostabilization, hyperaccumulation and dendroremediation. It focused on improving plant capacity to uptake translocate, stabilize, store and remove specific contaminants from polluted water and soil environments. Potential genes were identified and isolated from microbes and plants and manipulated for enhancing phytoremediation efficiency of plants and thus increase their survival percentage, tolerance level, storage and toxicant degradation capacity. 
Practical application of omics in phytoremediation technology has been represented in the Table 1. Integrated omics approaches may reveal information that remains undetected when organisms are examined in isolation as cited by Bell et al. ${ }^{[15]}$. Different metaorganism subcomponents like microbiome, interactome and plantome contribute to different properties of phytoremediation such as Relationships between gene content, expression, translation, and activity, Intermicrobial interactions, Relative plant:microbe investment in phytoremediation activity, Interkingdom (microbe to plant) gene transfer, Interspecies variability in gene content, expression, and activity, Root exudation patterns, Physiological responses across heterogeneous environments, Physiological responses across contaminant gradients, Physiological responses across heterogeneous environments and Variations in expression between tissues by integrating omics with traditional approach $^{[15]}$.

Multiple scale applications of Omics technologies are used in recent phytoremediation of soil contaminants through plants and microbes. Single organism omics involved cultured microorganisms, Isolated single cells of uncultivated microorganisms, plants. In cultured microorganisms approach complete biotransformation pathways or contaminant tolerant genes can be revealed from their genome assembly of isolate ${ }^{[15]}$. In this context, transcriptomic or proteomic approaches are able to identify contaminant tolerant genes or plant metabolites. Omics approach characterize gene, protein, and metabolite in plants, that reveals interplant variability thereby assist in plant selection and cultivation efforts for phytoremediation of contaminants. Mixed microbial communities and Metaorganism omics are involved in multiple-organism omics approach ${ }^{[15]}$.

Different bioinformatics tools those are used for implementation of Bioremediation technology are listed in Table 2.

Different bioinformatic approaches can be effectively used in tracing numerous biodegradation pathways, physiochemical analysis of toxins, prediction of thee levels of toxicity, genomic and proteomic approaches to enzyme system of microbes, prediction of path of degradation , and reaction kinetics for remediation of contaminants. So Bioinformatics hold prospective purpose for the development of phytoremediation technology ${ }^{[16]}$. Bioinformatics requires the study of plant genomics, proteomics, systems biology, computational biology, phylogenetic trees, data mining and application of major bioinformatics tools for

Table 1. Different Omics approaches, their purpose and instances in context of phytoremediation technology

\begin{tabular}{|c|c|c|}
\hline Omics approach & Purpose & Instances \\
\hline $\begin{array}{l}\text { Comparison of plant genomes with } \\
\text { phytoremediation efficiency to identify } \\
\text { optimal/novel targets for transgenes }\end{array}$ & To Improve genetic engineering of plants & $\begin{array}{c}\text { Identified genes potentially involved in the } \\
\text { detoxification of xenobiotic (RDX) pollutants via } \\
\text { transcriptomics }\end{array}$ \\
\hline Efficient isolation of remediation related genes & $\begin{array}{l}\text { High throughput sequencing of functional } \\
\text { metagenomic libraries }\end{array}$ & Review of function \\
\hline $\begin{array}{l}\text { Determine efficacy of soil amendments } \\
\text { Apply amendments over a wide range of } \\
\text { environmental conditions and compare } \\
\text { omics and functional responses to determine } \\
\text { parameters of efficiency Amplicon }\end{array}$ & $\begin{array}{l}\text { targeted metagenomic sequencing showed } \\
\text { that diesel degradation efficiency following } \\
\text { monoammonium phosphate addition was } \\
\text { correlated to organic matter content and } \\
\text { promotion of Betaproteobacteria }\end{array}$ & $\begin{array}{l}\text { Determine efficacy of soil amendments Apply } \\
\text { amendments over a wide range of environmental } \\
\text { conditions and compare omics and functional } \\
\text { responses to determine parameters of efficiency } \\
\text { Amplicon }\end{array}$ \\
\hline
\end{tabular}

Table 2. Different bioinformatics database and their principle of use in bioremediation technology (Source- Khan 2018)

\begin{tabular}{cc}
\hline Bioinformatics databases and tool & Use in bioremediation \\
\hline $\begin{array}{c}\text { AutoDock/AutodockVina, DOCK, DockoMatic, FINDSITE LHM, GalaxyDock, } \\
\text { GlamDock, Glide, GOLD, GriDock, Haddock, HomDock, Rosetta Ligand, } \\
\text { LeadFinder, Molegro Virtual Docker, PAR-3D, ParaDockS, PROCAT, Pocket-finder, } \\
\text { rDock, VinaMPI, YASARA Structure }\end{array}$ & Protein-ligand docking tool \\
BioCarta and WIT, Biocyc BRENDA, EcoCycsystem, ExPASy, KEGG, MetaCyc, \\
MetaRouter, PANTHER, Roche Biochemical Pathways UM-BBD \\
ChemDplus, Chemogenesis, PubChem \\
ACD / TOx suite, CAESAR,Comparative Toxicogenomics Database ECOSAR, \\
ECOTOX, GENE-TOX, \\
Hazard Expert, PBT profiler, Toxicity Estimation Tool (TEST) \\
Database of Biodegradative Oxygenases, KEGG \\
Phylemon2, Phylip, Phylodendron, PHYML, T-Rex & Metabolic pathways databases \\
\end{tabular}


determining the structures and biodegradative pathways of xenobiotic compounds ${ }^{[4]}$.

Insertion mutagenesis involving populations of T-DNA is one such Molecular genetics approaches, which can be used to identify genes for hyper-accumulation ${ }^{[17]}$. Recently, considerable progress has been made though genomics study for identifying metal ion transporter plant genes ${ }^{[18,19]}$. Phytoremediation technology is still in its premature development stages which have just indicated efficiency of the plants for toxic metal remediation. Public awareness about this omics approach for phytoreemediation technology is considered and clear and precise information is made available to the general public to augment its suitability as a global sustainable technology for wide use ${ }^{[20]}$. Recently a new concept on use of symbiotic proteomics tools for better understanding of the molecular bases of cell communication and the regulation of developmental and metabolic pathways in mycorrhizal associations were established for general increase in stress tolerance and health. Chapelo et al. ${ }^{[21]}$ studied that proteomics gives a better understanding to identify proteins expressed and regulated during the development and functioning of mycorrhizal symbioses, and thus contribute in information for events occurring at the cellular level

The need of the hour is implementation and popularization of proteomics and bioinformatics based phytoremediation technology which is considered as one of the recent biotechnological advancement in this field.

\section{Conclusions}

The combined science of biology and information technology i,e, called Bioinformatics basically focuses on the biotechnological application at cellular and molecular levels for. Phytoremediation is the novel technology of employing plant's potentiality for degradation of toxic contaminants in soil and water. Different plants display an amazing array of contaminant degradation ability that can efficiently and effectively re-establish a sustainable environment. In this regard different aspects of genomics and proteomics have proved effective in phytoremediation studies. The revolutionary role of proteomics and bioinformatics in phytoremediation of contaminants will be an effective approach for combating environmental stress. Proteomics and bioinformatics based phytoremediation is considered and could be named as smart phytoremediation technology employed for quick recuperation of environment from pollutants and wastes. This smart technology provides insights into the complex behavior of enzymes, proteins and metabolites action and their biochemical pathways for degradation of wastes. This leads towards deriving a sustainable solution for environmental pollution.

\section{References}

[1] Mohanty M. (2016), 'Post-Harvest Management of Phytoremediation Technology", J Environ Anal Toxicol, 2016, 6:5, 1-8.

[2] Zhang, C, Bennett GN (2005), "Biodegradation of xenobiotics by anaerobic bacteria", Appl Microbiol Biotechnol, 67: 600-618.

[3] Samanta, SK., Singh, O.V. and Jain, R.K. (2002), "Polycyclic aromatic hydrocarbons: environmental pollution and bioremediation", Trends Biotechnol, 20: 243-248.

[4] Fulekar, M. H., \& Jaya, S. (2008), "Bioinformatics for bioremediation", Innovative Romanian Food Biotechnology, 2(2)28-36.

[5] Coleman, J. O., Haslam, R. P., \& Downie, A. L. (2005), "Transcriptomics and proteomics: Tools for optimising phytoremediation activities", Zeitschrift für Naturforschung $C$, 60(7-8), 544-548.

[6] Leung M (2004), "Bioremediation: techniques for cleaning up a mess", J Biotechnol, 2:18-22.

[7] Wojas S, Hennig J, Plaza S, Geisler M, Siemianowski O et al (2009), "Ectopic expression of Arabidopsis ABC transporter MRP7 modifies cadmium root-toshoot transport and accumulation", Environ Pollut, 157(10):2781-2789.

[8] Singh. JS, Abhilash PC, Singh HB, Singh RP, Singh DP (2011), "Genetically engineered bacteria: an emerging tool for environmental remediation and future research perspectives" Gene, 480:1-9.

[9] Dhankher OP, Doty SL, Meagher RB, Pilon-Smits E (2011), "Biotechnological approaches for phytoremediation", In: Altman A, Hasegawa PM (eds) Plant biotechnology and agriculture. Academic Press, Oxford, pp 309-328.

[10] Kumar C, Mani D (2012), "Advances in bioremediation of heavy m(etals: a tool for environmental restoration", LAP LAMBERT Academic Publishing A)-G \& Co. KG, Saarbru"cken.

[11] Mani D, Kumar, C. (2014), "Biotechnological advances in bioremediation of heavy metals contaminated ecosystems: an overview with special reference to phytoremediation", Int. J. Environ. Sci. Technol, 11:843-872.

[12] Lyyra S, Meagher RB, Kim T, Heaton A et al (2007), "Coupling two mercury resistance genes in Eastern cottonwood enhances the processing of organomercury", Plant Biotechnol J, 5:254-262.

[13] Bañuelos, G., Terry, N., LeDuc, D. L., Pilon-Smits, E. A., \& Mackey, B. (2005), "Field trial of transgenic Indian mustard plants shows enhanced phytoremedi- 
ation of selenium-contaminated sediment", Env. Sci. \& Techn., 39(6), 1771-1777.

[14] Poirier I, Hammann P, Kuhn L, Bertrand M (2013), "Strategies developed by the marine bacterium Pseudomonas fluorescens BA3SM1 to resist metals: a proteome analysis", Aquat Toxicol, 128(129), 215232.

[15] Bell TH, Joly S, Pitre FE , and Yergeau E (2014), "Increasing phytoremediation efficiency and reliability using novel omics approaches", Trends in Biotech, $32,(5), 271-280$.

[16] Khan N.T.(2018), "Integration of Bioinformatics in Bioremediation", Int J Biomed Data Min 7, 1000130. DOI: $10.4172 / 2090-4924.1000130$.

[17] Pollard AJ, Powell KD, Harper FA, Smith JAC (2002), "The genetic basis of metal hyperaccumulation in plants", Crit. Rev. Plant Sci, 21,539-566.

[18] Papoyan A, Kochian LV (2004), "Identification of Thlaspi caerulescens genes that may be involved in heavy metal hyperaccumulation and tolerance-Characterization of a novel heavy metal transporting AT-
Pase". Plant Physiol, 136, 3814-3823.

[19] Weber M, Harada E, Vess C, von Roepenack-Lahaye E, Clemens S (2004), "Comparative microarray analysis of Arabidopsis thaliana and Arabidopsis halleri roots identifies nicotianamine synthase, a ZIP transporter and other genes as potential metal hyperaccumulation factors", Plant J, 37,269-281.

[20] Gratão, P. L., Prasad, M. N. V., Cardoso, P. F., Lea, P. J., \& Azevedo, R. A. (2005), "Phytoremediation: green technology for the cleanup of toxic metals in the environment”, Braz J Plant Physiol, 17(1), 53-64.

[21] Chiapello, M., Perotto, S., \& Balestrini, R. (2015), "Symbiotic Proteomics-State of the art in plant-mycorrhizal fungi interactions", Recent Adv in Proteomics Res, 10, 61331.

[22] Martins, I.J. (2016) Anti-Aging Genes Improve Appetite Regulation and Reverse Cell Senescence and Apoptosis in Global Populations. Advances in Aging Research, 5, 9-26. 